\title{
Influence of linear and non-linear constitutive models on thermoacoustic waves in an enclosure
}

\author{
L. Škerget \& J. Ravnik \\ University of Maribor, Faculty of Mechanical Engineering, \\ Smetanova ulica 17, SI-2000 Maribor, Slovenia
}

\begin{abstract}
The problem of unsteady compressible fluid flow in an enclosure induced by thermoacoustic waves is studied numerically. Full compressible set of Navier-Stokes equations are considered and numerically solved by boundary-domain integral equations approach, coupled with wavelet compression and domain decomposition to achieve numerical efficiency. The thermal energy equation is written in its most general form including the Rayleigh and reversible expansion rate terms. Both the classical Fourier heat flux model and wave heat conduction model are investigated. The momentum flux is modelled using standard Newtonian viscous model and linear viscoelastic Maxwell model.

The velocity-vorticity formulation of the governing Navier-Stokes equations is employed, while the pressure field is evaluated from the corresponding pressure Poisson equation. Material properties are taken to be for the perfect gas and assumed to be pressure and temperature dependent.

Keywords: compressible fluid flow, velocity-vorticity formulation, Navier-Stokes equations, thermoacoustic waves.
\end{abstract}

\section{Introduction}

In the paper the generation and transmission of thermoacoustic waves in an perfect gas filled closed cavity is studied numerically. When a compressible fluid is subjected to a rapid increase in temperature at a solid wall, a sudden expansion of the adjacent gas occurs. This phenomenon generates a fast increase in the local pressure and leads to the production of pressure waves. These thermally generated waves are referred to as thermoacoustic waves.

Thermoacoustic transport phenomena may be very interesting, when the fluid is close to thermodynamic critical point or when other modes of transport mechanism 
are weak or absent. Thermoacoustic waves enhance heat transport by converting the thermal energy into compression work and by inducing convection motion away from the heated wall. This mode of heat transfer is dominant in the space environment under zero- or reduced-gravity conditions, where natural convection of heat transport may be absent or low, and when heat conduction is the only mode of heat transport mechanism.

The problem of thermoacoustic transport phenomena in a perfect gas confined in an enclosure subjected to a sudden increase in temperature at a solid wall, have been considered experimentally and numerically by Huang and Bau [1] as well as by Brown and Churchill [2]. In the literature one can find analytical attempts to study the phenomenon limited mainly to simplified models, while the solutions to general cases have been performed by numerical approximative methods. In the present paper the boundary element method (BEM) is used. Previous studies of Lin and Farouk [3], Aktas and Farouk [4], Škerget and Ravnik [5], observed strong thermoacoustic waves as a consequence of impulsive heating. Horizontal velocity component reverses sign after reflection from the side walls. The flow field is essentially one-dimensional.

\section{Governing equations for the primitive variables formulation}

The field functions of interest are velocity vector field $v_{i}$, scalar pressure field $p$, temperature field $T$ and the field of mass density $\rho$, so that the mass, momentum and energy equations are given by the following set of nonlinear equations:

$$
\begin{aligned}
\frac{\partial v_{j}}{\partial x_{j}}= & \mathcal{D}=-\frac{1}{\rho} \frac{D \rho}{D t} \\
\rho \frac{D v_{i}}{D t}= & -e_{i j k} \frac{\partial \eta \omega_{k}}{\partial x_{j}}+2 e_{i j k} \frac{\partial \eta}{\partial x_{j}} \omega_{k}+2 \frac{\partial \eta}{\partial x_{j}} \frac{\partial v_{i}}{\partial x_{j}} \\
& +\frac{4}{3} \frac{\partial \eta \mathcal{D}}{\partial x_{i}}-2 \mathcal{D} \frac{\partial \eta}{\partial x_{i}}-\frac{\partial p}{\partial x_{i}}+\rho g_{i}, \\
c \frac{D T}{D t}= & \frac{\partial}{\partial x_{j}}\left(k \frac{\partial T}{\partial x_{j}}+\lambda \frac{\partial q_{j}}{\partial t}\right)+\beta T \frac{D p}{D t}+\Phi,
\end{aligned}
$$

in the Cartesian frame $x_{i}$, where $c$ denotes changeable isobaric specific heat capacity per unit volume, $c=c_{p} \rho, t$ is time, $g_{i}$ is gravitational acceleration vector, while $\beta$ is a volume coefficient of thermal expansion, $\lambda$ is a heat relaxation time and $\Phi$ is the Rayleigh viscous dissipation function. Because of the analytical reasons required by the derivation of the velocity-vorticity formulation of the governing equations, the momentum equation is given in the second extended form.

The Newton momentum viscous and Maxwell viscoelastic constitutive models are considered, such as

$$
\tau_{i j}=\tau_{i j}^{v}+\tau_{i j}^{e}=2 \eta \dot{\varepsilon}_{i j}-\frac{2}{3} \eta \mathcal{D} \delta_{i j}-\lambda_{1} \frac{\partial \tau_{i j}}{\partial t},
$$


where the extra stress tenzor or the momentum flux tenzor is given as a sum of viscous $\tau_{i j}^{v}$ and elastic $\tau_{i j}^{e}$ effects. $\mathcal{D}=\operatorname{div} \vec{v}=\dot{\varepsilon}_{i i}$ represents the divergence of the velocity field or local expansion field. The parameter $\lambda_{1}$ is the stress relaxation time. The Rayleigh dissipation function may be stated as

$$
\Phi=\tau_{i j} \frac{\partial v_{i}}{\partial x_{j}}=\eta\left(\frac{\partial v_{i}}{\partial x_{j}} \frac{\partial v_{i}}{\partial x_{j}}+\frac{\partial v_{j}}{\partial x_{i}} \frac{\partial v_{j}}{\partial x_{i}}\right)-\frac{2}{3} \eta \mathcal{D}^{2} .
$$

In the case of intensive unsteady heat transfer, it is important to take into account a terminal velocity of a moving temperature frontier, namely the following form of heat flux constitutive model

$$
q_{i}=-k \frac{\partial T}{\partial x_{i}}-\lambda \frac{\partial q_{i}}{\partial t}
$$

where material constants $k$ and $\lambda$ are the heat conductivity and the heat relaxation time. For most heat transfer problems of practical importance, the simplification known as the Fourier law of heat diffusion is accurate enough, namely $q_{i}=-k \frac{\partial T}{\partial x_{i}}$.

Representing the material properties of the fluid the dynamic viscosity $\eta$, heat conductivity $k$, the specific heat per unit volume $c$, and the mass density $\rho$, are written as sums of a constant and variable part, e.g. $\eta=\eta_{o}+\tilde{\eta}, k=k_{o}+\widetilde{k}$, $c=c_{o}+\widetilde{c}$, and $\rho=\rho_{o}+\widetilde{\rho}$, so the momentum and energy eqs. (2) and (3) may be written in analogy to the basic conservation equations formulated for the constant material properties

$$
\begin{aligned}
\frac{D \vec{v}}{D t} & =-v_{o} \vec{\nabla} \times \vec{\omega}-\frac{1}{\rho_{o}} \vec{\nabla} p+\frac{\rho}{\rho_{o}} \vec{g}+\frac{1}{\rho_{o}} \vec{f}^{m}+\frac{1}{\rho_{o}} \vec{\nabla} \cdot \underline{\tau}^{e}, \\
\frac{D T}{D t} & =a_{o} \Delta T+\frac{S^{m}}{c_{o}},
\end{aligned}
$$

where the pseudo body force term $\vec{f}^{m}$ and pseudo heat source term $S^{m}$ are introduced into the momentum eq. (7) and into energy eq. (8) respectively, capturing the variable material property effects, and the nonlinear effects due to rate of reversible and irreversible work, and given by expressions, e.g. for plane flow problems

$$
f_{i}^{m}=-e_{i j} \frac{\partial \tilde{\eta} \omega}{\partial x_{j}}+2 e_{i j} \frac{\partial \widetilde{\eta}}{\partial x_{j}} \omega+2 \frac{\partial \eta}{\partial x_{j}} \frac{\partial v_{i}}{\partial x_{j}}+\frac{4}{3} \frac{\partial \eta \mathcal{D}}{\partial x_{i}}-2 \mathcal{D} \frac{\partial \eta}{\partial x_{i}}-\tilde{\rho} a_{i},
$$

while the pseudo heat source term is given by an expression

$$
S^{m}=\vec{\nabla}\left(\widetilde{k} \vec{\nabla} T+\lambda \frac{\partial \vec{q}}{\partial t}\right)-\widetilde{c} \frac{D T}{D t}+\beta T \frac{D p}{D t}+\Phi,
$$

in which the kinematic viscosity is $v_{o}=\eta_{o} / \rho_{o}$, the heat diffusivity $a_{o}=k_{o} / c_{o}$ and the inertia acceleration vector is $\vec{a}=D \vec{v} / D t$. 


\section{Governing equations for the velocity-vorticity formulation}

The kinematics of the flow motion is governed by the following vector elliptic Poisson equation for the velocity vector

$$
\nabla^{2} \vec{v}+\vec{\nabla} \times \vec{\omega}-\vec{\nabla} \mathcal{D}=0
$$

The kinetics of the flow representing the vorticity transport equation is obtained by applying the curl differential operator to the both sides of eq. (7), rendering the following statement for the two-dimensional plane flow written in Cartesian tensor notation form as

$$
\frac{\partial \omega}{\partial t}+\frac{\partial v_{j} \omega}{\partial x_{j}}=v_{o} \frac{\partial^{2} \omega}{\partial x_{j} \partial x_{j}}-\frac{1}{\rho_{o}} e_{i j} \frac{\partial \rho g_{i}}{\partial x_{j}}-\frac{1}{\rho_{o}} e_{i j} \frac{\partial f_{i}^{m}}{\partial x_{j}}-\frac{1}{\rho_{o}} e_{i j} \frac{\partial}{\partial x_{j}}\left(\frac{\partial \tau_{i k}^{e}}{\partial x_{k}}\right) .
$$

To derive the pressure equation, depending on known velocity field, vorticity field and material functions, the divergence of momentum equation should be calculated, resulting in the elliptic Poisson pressure equation

$$
\Delta p-\vec{\nabla} \cdot \vec{f}_{p}=0,
$$

where the pressure force term $\vec{f}_{p}$ is for the planar flow cases

$$
\frac{\partial p}{\partial x_{i}}=f_{p i}=-\eta_{o} e_{i j} \frac{\partial \omega}{\partial x_{j}}-\rho_{o} a_{i}+\rho g_{i}+f_{i}^{m}+\vec{\nabla} \cdot \underline{\tau}^{e} .
$$

The Neumann boundary conditions for pressure equation may be determined for the whole solution domain and the following relation is valid

$$
\frac{\partial p}{\partial n}=\vec{f}_{p} \cdot \vec{n} \quad \text { on } \quad \Gamma \text {. }
$$

Due to the variable material property terms, and rate of reversible and irreversible work acting as additional temperature, pressure and velocity field dependent source terms, the vorticity, thermal energy, and pressure equations are coupled, making the numerical solution procedure of this highly nonlinear coupled set of equations very severe. Already, the vorticity transport equation as itself is highly nonlinear partial differential equation due to the inherent nonlinearity caused by the compatibility and restriction conditions among velocity, vorticity and dilatation fields. The dilatation and the vortical part of the flow, $\mathcal{D}$ and $\vec{\omega}$ field functions respectively, and all other nonlinear terms have to be under-relaxed to achieve the convergence of the numerical solution procedure.

\section{Numerical algorithm}

The nonlinear system of partial differential equation described in the previous section is solved by a combination of two BEM techniques. The wavelet compressed BEM (Ravnik et al. [6]) is used to calculated boundary values of vorticity and 
pressure, while a macro element BEM governed by a square system matrix is used to solve other equations. The algorithm is presented in detail below:

- calculate integrals, that depend solely on the mesh geometry

- use wavelet compression on matrices of integrals

- begin time step loop

- begin global nonlinear loop

- begin local kinematic-vorticity loop

- KINEMATICS

* calculate boundary values by solving the kinematics equation by wavelet compressed single domain BEM

* calculate domain velocity values by solving the kinematics equation by subdomain BEM

- VORTICITY KINETICS

* solve vorticity transport equation by subdomain BEM using the boundary values from single domain BEM calculation

* check convergence - repeat steps in the local loop until convergence is achieved

- end local kinematic-vorticity loop

- ENERGY KINETICS

* temperature

- PRESSURE COMPUTATION

* calculate boundary values by solving the pressure equation by wavelet compressed single domain BEM

* calculate domain pressure values by solving the pressure equation by subdomain BEM

- NON-LINEAR EFFECTS

* calculate all nonlinear terms (dilatation, reversible and irreversible rate of work, nonlinear material properties)

- CONSTITUTIVE FLUX MODELS

* Fourier and non-Fourier heat flux model

* Newton viscous and Maxwell viscoelastic models

* check convergence - repeat steps in the nonlinear loop until convergence is achieved

- end global nonlinear loop

- end time step

- output results

With the aim of decreasing storage and CPU time requirements of the single domain BEM we employ the macro element approach. The idea is to use a collocation scheme for equation for each domain cell separately and require that the functions must be continuous over the domain cell boundaries. Since every domain cell neighbours only to a few cells, we end up with a sparse system of equations. In a nutshell we are using single domain BEM on every domain cell separately and connect them via compatibility and equilibrium conditions (Škerget et al. [7], Ravnik et al. [8]). 
In order to be able to perform simulations on dense grids, we used a wavelet compression algorithm on fully populated matrices, resulting from the BEM calculation of boundary vorticity. A discrete wavelet transform for vectors of arbitrary length, developed by Ravnik et al. [6, 9], was used.

\section{Numerical example}

Using the developed numerical scheme we analysed the onset of thermoacoustic waves in an enclosure. The enclosure is $13 \mathrm{~mm}$ wide and $13 \mathrm{~mm}$ high. It is filled with nitrogen gas at $p=101325 \mathrm{~Pa}$ and $T_{0}=300 \mathrm{~K}$. In the beginning the fluid is at rest. At $t=0$ the left wall is impulsively heated to $T_{L}=400 \mathrm{~K}$. During the simulation $(t>0)$ a constant temperature of $T_{R}=300 \mathrm{~K}$ is kept on the right wall and $T_{L}=400 \mathrm{~K}$ on the left. The top and bottom walls of the enclosure are considered adiabatic, i.e. there is no heat flux through them. Gravity is neglected. The enclosure is closed by walls, which have a no-slip velocity boundary condition.

The ideal gas is chosen as a working fluid, thus the equation of state for the perfect gas is applied

$$
p=\rho R T,
$$

to relate the temperature to the other thermodynamic quantities. In the present research the Prandtl $(\mathrm{Pr})$ number is assumed to remain constant, e.g. the following constant physical values are selected for the nitrogen $\left(N_{2}\right): \kappa=1.4, R=296.7$ and $\operatorname{Pr}=0.713$. The temperature dependence of the material properties are taken into account using the following polynomial expressions, e.g. for the dynamic viscosity $\eta$

$$
\eta(T)=-1.253 \cdot 10^{-6}+8.983 \cdot 10^{-8} T-1.139 \cdot 10^{-10} T^{2}+9.101 \cdot 10^{-14} T^{3},
$$

the heat conductivity

$$
k(T)=1.494 \cdot 10^{-4}+1.108 \cdot 10^{-4} T-1.045 \cdot 10^{-7} T^{2}+6.958 \cdot 10^{-11} T^{3},
$$

while the heat relaxation time value $\lambda=0.1$ was selected. The influence of temperature on $c_{p}$ is neglected.

For the impulsive heating, the temperature of the left wall is given as

$$
T_{L}(t)=T_{0}(1+A),
$$

where the over heat ratio $A$ is given by an expression

$$
A=\frac{T_{L}-T_{0}}{T_{0}},
$$

and $A=1 / 3$ for all cases presented for spatially uniform wall heating, thus the left wall temperature is equated to $T_{L}=400 \mathrm{~K}$.

Three meshes were used in simulations. The coarse mesh with $20 \times 20$ elements (1681 nodes), the middle mesh with $30 \times 30$ elements $(3721$ nodes) and the fine 

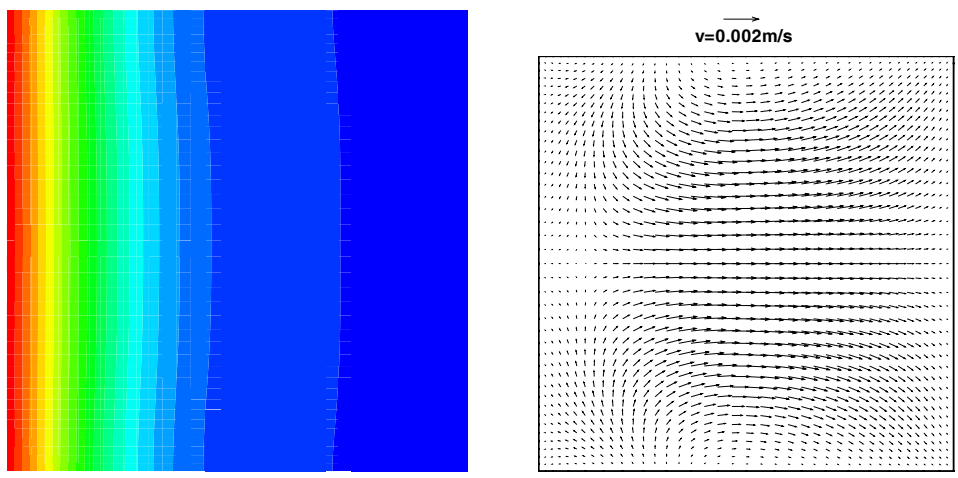

Figure 1: Temperature contours (left) and velocity vectors (right) at $t=0.15 \mathrm{~s}$, zero gravity condition, $20 \times 20$ element mesh, $\Delta t=10^{-4} \mathrm{~s}$.

mesh with $40 \times 40$ elements ( 6561 nodes). Two time steps were used for simulation. The long time step of $\Delta t=10^{-4} \mathrm{~s}$, which enabled a long-term simulation reaching steady state. The short time step of $\Delta t=10^{-6} \mathrm{~s}$ enabled an insight into the physics of the phenomenon. The short time step was used on the coarse and middle meshes, while the long time step was employed on the fine mesh.

The short time step simulation lasted for $0.8 \mathrm{~s}$. On Figure 1 we are showing the temperature field and the velocity fields at $t=0.15 \mathrm{~s}$. Since there is no gravity and hence no buoyancy the flow field appears to be one-dimensional.

A point in the middle of the enclosure $(x=0.065 \mathrm{~m}, y=0.065 \mathrm{~m})$ was chosen for demonstration of the time development of the flow. On Figure 2 (left) a pressure and temperature evolution with time is shown. We observe a gradual increase of pressure, faster in the first tenth of a second and slower after that. No pressure waves are observed, which was expected since the time step is too long to capture phenomena which occur with the speed of sound. The pressure distribution in the
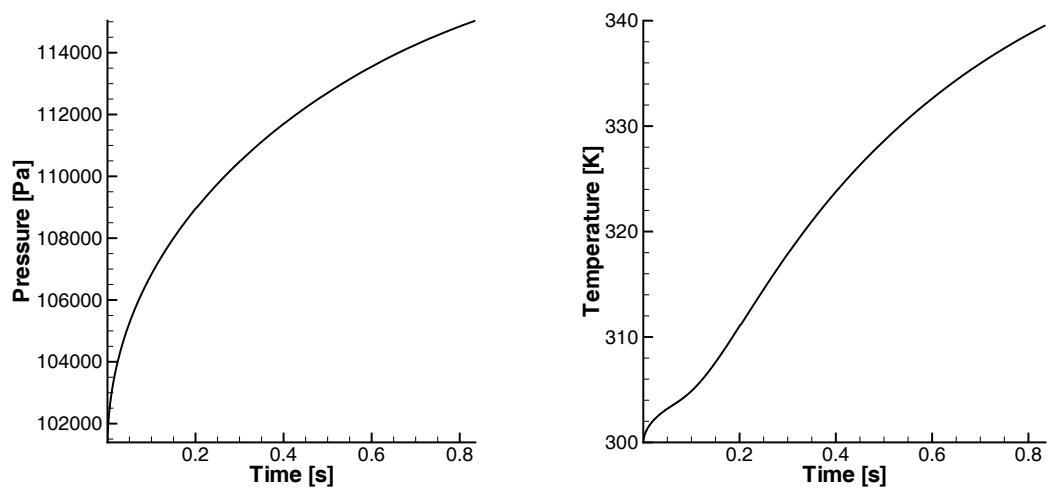

Figure 2: Pressure (left) and temperature (right) in the centre of the enclosure ( $x=$ $0.065 \mathrm{~m}, y=0.065 \mathrm{~m}$ ) versus time; $\Delta t=10^{-4} \mathrm{~s}$. 

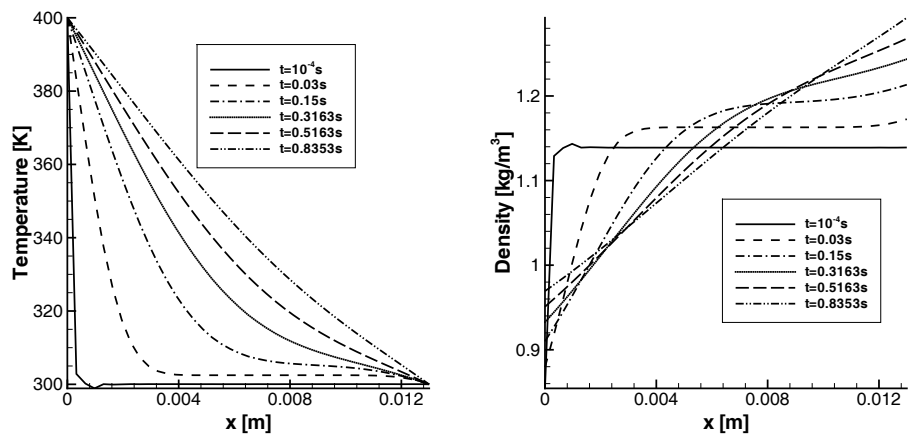

Figure 3: Temperature (left) and density (right) profiles through the centreline of the cavity. Development of the profile with time can be seen; $\Delta t=$ $10^{-4} \mathrm{~s}$.

enclosure is uniform. At $t=0.8 \mathrm{~s}$ the pressure reaches about $p=114000 \mathrm{~Pa}$. Looking at the evolution of the temperature of nitrogen in the middle of the enclosure on Figure 2 (right) we observe that the temperature increase is more or less linear. The nitrogen in the centre of the enclosure reaches $T=340 \mathrm{~K}$.

To show the variation of flow fields across the enclosure we examined profiles along the horizontal centreline $(x, 0.065 \mathrm{~m})$. Figure 3 shows temperature and density profiles for six time instants. The very sharp temperature profile in the beginning of the simulation is caused by the application of boundary conditions. At long times, a steady state diffusive temperature profile is observed. Since the ideal gas approximation of nitrogen is assumed and since the pressure distribution in the cavity is uniform the density profiles are reciprocal to those of temperature.

The simulation on the fine mesh was preformed using a time step of $10^{-6} \mathrm{~s}$. Figure 4 shows the time evolution of pressure and horizontal velocity component
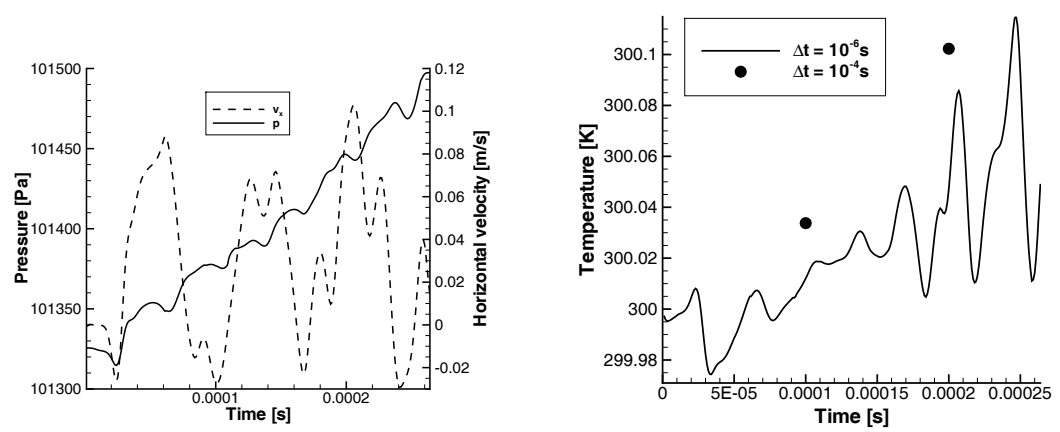

Figure 4: Pressure and horizontal velocity in the centre of the enclosure with time (left); Temperature in the centre of the enclosure with time (right); $40 \times$ 40 element mesh, $\Delta t=10^{-6} \mathrm{~s}$. 

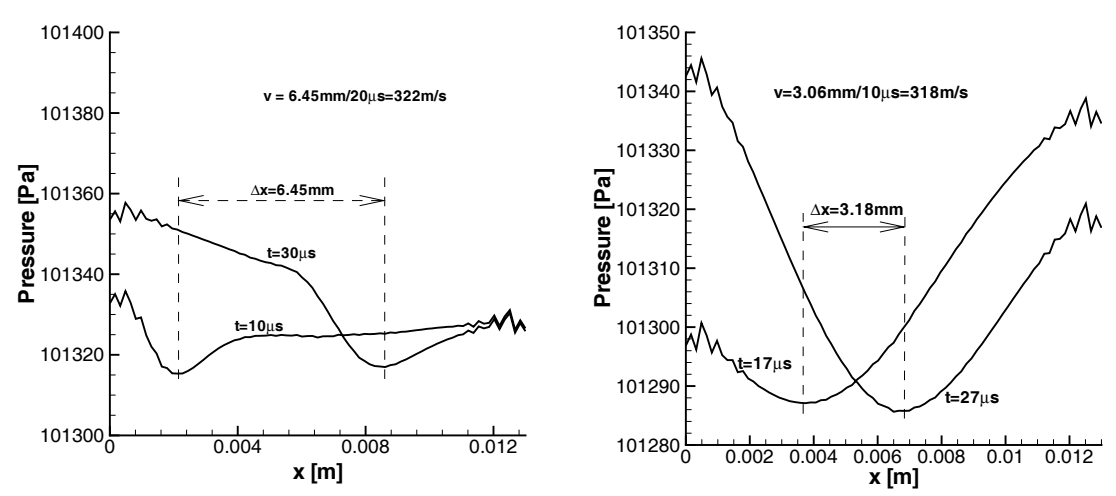

Figure 5: Pressure profiles through the centre of the enclosure; $40 \times 40$ element mesh, $\Delta t=10^{-6} \mathrm{~s}$. Left Fourier heat diffusion rheological model, right non-Fourier heat diffusion rheological model.

in the centre of the enclosure. Due to the presence of thermoacoustic waves a fluctuating increase of pressure and changing sign of horizontal velocity is observed. At the same time a fluctuating increase of temperature is also observed.

Figure 5, left, shows pressure profiles across the centreline of the enclosure at $t=10 \mu \mathrm{s}$ and $t=30 \mu \mathrm{s}$. By measuring the distance travelled by the thermoacoustic wave between these time instants we were able to estimate the speed of sound to about $322 \mathrm{~m} / \mathrm{s}$, which is within $10 \%$ of the correct value for nitrogen.

The non-Fourier heat transfer model was also simulated on the fine mesh with a time step of $\Delta t=10^{-6} \mathrm{~s}$. Using subsequent pressure profiles we were able to estimate the distance travelled by the pressure wave in a specified time interval. This enabled the estimation of the speed of sound, which amounted to about $318 \mathrm{~m} / \mathrm{s}$. The two profiles were selected to be $10 \mu$ s apart and are displayed on Figure 5, right.

\section{Conclusions}

In this work a numerical algorithm for solving laminar compressible viscous flow and heat transfer is presented. The method is used to simulate the onset of thermoacoustic waves in a nitrogen filled enclosure subjected to a high temperature gradient. The results show the occurrence of thermoacoustic waves travelling across the enclosure at the speed of sound. During the expansion phase, the wave temperature dips bellow the average medium temperature. The thermoacoustic waves propagate at approximately the speed of sound within the fluid and gradually damp out due to heat and momentum diffusion. Extremely high gradients are present in the flow field, which generate the pressure waves and are difficult to model numerically. Our findings are consistent with numerical and experimental results of other authors, confirming the applicability of BEM for such flows. 
Detailed investigation of the influence of different constitutive models on the flow phenomenon will be presented at the conference.

\section{References}

[1] Huang, Y. \& Bau, H., Thermoacoustic waves in a confined medium. Int J Heat Mass Transfer, 40, pp. 407-419, 1997.

[2] Brown, M. \& Churchill, S., Experimental measurements of pressure waves generated by impulsive heating of a surface. AIChE Journal, 41, pp. 205-213, 1995.

[3] Lin, Y. \& Farouk, B., Flows induced by thermoacoustic waves in an enclosure: efffect of gravity. J Thermophys Heat Transfer, 20, pp. 376-383, 2006.

[4] Aktas, M. \& Farouk, B., Numerical simulation of developing natural convection in an enclosure due to rapid heating. Int J Heat Mass Transfer, 46, pp. 2253-2261, 2003.

[5] Škerget, L. \& Ravnik, J., Numerical simulation of compressible fluid flow in an enclosure induced by thermoacoustic waves. Numerical analysis and applied mathematics, (AIP Conference Proceedings, 936), ed. T. Simos, American Institute of Physics, pp. 523-526, 2007.

[6] Ravnik, J., Škerget, L. \& Hriberšek, M., The wavelet transform for BEM computational fluid dynamics. Eng Anal Bound Elem, 28, pp. 1303-1314, 2004.

[7] Škerget, L., Hriberšek, M. \& Žunič, Z., Natural convection flows in complex cavities by BEM. Int J Num Meth Heat \& Fluid Fl, 13, pp. 720-735, 2003.

[8] Ravnik, J., Škerget, L. \& Žunič, Z., Combined single domain and subdomain BEM for 3D laminar viscous flow. Eng Anal Bound Elem, submitted, 2008.

[9] Ravnik, J., Škerget, L. \& Hriberšek, M., 2D velocity vorticity based LES for the solution of natural convection in a differentially heated enclosure by wavelet transform based BEM and FEM. Eng Anal Bound Elem, 30, pp. 671686, 2006. 